\title{
Sincronização por Realimentação de Erro no Circuito Eletrônico da Partícula em Mesa Vibratória
}

\section{Synchronization by Error Feedback in an Electronic Particle Circuit on Vibratory Table}

\author{
José Carlos Pizolato Junior ${ }^{1}$, Carlos Alberto De Franciscoํㅜ, Taufik Abrão²
}

\begin{abstract}
Resumo
Este trabalho analisa pela primeira vez o sincronismo e o controle caótico aplicados ao circuito eletrônico da partícula em mesa vibratória. Esta análise foi realizada a partir da implementação da técnica de sincronismo por realimentação de erro em sistemas de comunicação caóticos. A robustez do acoplamento entre os dois sistemas foi verificada experimentalmente nas condições de descasamento de parâmetros (sinal senoidal e sinal DC). Resultados experimentais comprovam a robustez destes sistemas em aplicações com requisitos de sincronismo entre transmissor e receptor.
\end{abstract}

Palavras-chave: Circuito eletrônico da partícula em mesa vibratória. Circuito caótico. Sincronismo.

\begin{abstract}
This paper examines at a first time and the chaotic control applied to the electronic particle circuit on the vibrating table. This investigation was carried out on the implementation of the timing error feedback technique in two chaotic systems. The strength of the coupling between both chaotic systems was verified experimentally in mismatch conditions parameters, e.g., sinusoidal and DC signal. Experimental results demonstrate the robustness of these systems in applications with timing requirements between transmitter and receiver.
\end{abstract}

Keywords: Electronic particle circuit in vibrating table. Chaotic circuit. Synchronization.

\footnotetext{
${ }^{1}$ Departamento de Engenharia Elétrica; Universidade Federal de São Carlos - UFSCar; São Carlos, Brasil jcpizolato@yahoo.com.br

${ }^{2}$ Docente do Departamento de Engenharia Elétrica da Universidade Estadual de Londrina; taufik@uel.br
} 


\section{Introdução}

Caos tem sido desenvolvido e muito estudado nas últimas duas décadas. Um sistema caótico é um sistema determinístico não-linear que apresenta um comportamento complexo e imprevisível. Este comportamento é sensivelmente dependente das variações das condições iniciais e parâmetros do sistema- Dentre os vários sistemas caóticos estudados na literatura destacam-se: implementação eletrônica do mapa logístico (SUNEEL, 2006), circuito eletrônico de Chua (CHUA et al., 1993), (LI et al., 2003), (WU et al., 2013), (FRADKOV; ANDRIEVSKY; EVANS, 2009), (FRADKOV; ANDRIEVSKY; EVANS, 2008), circuitos caóticos baseados em Lorenz (CUOMO; OPPENHEIM; STROGATS, 1993), (HUANG; ZHANG; ZHAO, 2015), (BOCHENG; ZHONG; JIANPING, 2009), (LI et al., 2014), (LIANG; ZHANG; XIA, 2008) circuitos eletrônicos Sprott (SPROTT, 2000), circuito eletrônico da partícula em caixa (PIZOLATO JUNIOR ; ROMERO; NETO, 2008), circuito caótico Jerk (SPROTT, 2011), (SRISUCHINWONG; SUKJARERNCHAIKUL, 2014), (LI; JIN; CHEN, 2014) e circuito eletrônico da particular em mesa vibratória (PFALTZGRAFF, 1969), (ZIMMERMAN; CELASCHI; NETO, 1992), (MELLO; TUFILLARO, 1987) e (JOSEPH; MARIÑO; SANJUÁN, 2012).

Outro aspecto importante sobre sistemas caóticos está no sincronismo entre eles. O fenômeno do sincronismo em sistemas caóticos acoplados tem sido muito estudado nas seguintes áreas e aplicações: dinâmica do laser, transmissão de informação por meio de sinais caóticos, sistemas biológicos, fisiológicos e circuitos eletrônicos. $\mathrm{Na}$ literatura, diferentes tipos de sincronização caótica entre osciladores acoplados, tais como sincronizações completa, generalizada e de fase têm sido descritas teoricamente e caracterizadas experimentalmente. Sincronização completa (CS) (PECORA; CARROLL, 1990) implica na coincidência de estados entre os sistemas em interação, $x_{1}(t)=x_{2}(t)$; este tipo somente aparece quando os sistemas que interagem forem idênticos. Caso contrário, se os parâmetros dos osciladores acoplados apresentarem um pequeno descasamento de parâmetros, então os estados estão próximos, isto é, $\left\|x_{1}(t)-x_{2}(t)\right\| \approx 0$, mas permanecem distintos (PIKOVSKY, 1984). A sincronização generalizada (GS) (RULKOV et al., 1995), (PISARCHIK; RUIZ-OLIVERAS, 2010), (HE et al., 2011), conhecida como sistema mestre-escravo, é definida como a presença de alguma relação funcional entre os estados dos sistemas mestre e escravo, isto é, $x_{2}(t)=F\left\{x_{1}(t)\right\}$. Finalmente, a sincronização de fase (PS) descrita em (ROSENBLUM; PIKOVSKY; KURTHS, 1997), significa coincidência de fases de osciladores caóticos, onde suas amplitudes permanecem caóticas e não correlacionadas.

Um simples circuito eletrônico que apresenta comportamento complexo é o circuito eletrônico da partícula em mesa vibratória. Ele foi introduzido por Fermi, e tem sido estudado experimentalmente e teoricamente (TUFILLARO; ALBANO, 1986), (VINCENT, 1995), (REIST; D'ANDREA, 2012). Este circuito emula eletronicamente o comportamento dinâmico do sistema mecânico de uma partícula colidindo com uma superfície em movimento. O comportamento caótico do circuito da partícula em mesa vibratória poderia ser aplicado para implementar um sistema de encriptação de dados análogo a outros trabalhos já apresentados (PIZOLATO JUNIOR; ROMERO; NETO, 2008). Neste caso, para um sistema de comunicação coerente, deve haver sincronismo entre sistemas transmissor e receptor. Estes sistemas são não-autônomos, ou seja, dependem de um sinal forçante que varia com o tempo. No contexto das telecomunicações por exemplo, sinais forçantes (sinais oscilantes) do transmissor e receptor devem estar tão próximos quanto possível, tendo em vista permitir sincronização, bem como obter uma qualidade de detecção coerente aceitável. Entretanto, os autores deste trabalho desconhecem qualquer investigação disponível na literatura especializada sobre o controle e sincronização no comportamento caótico do circuito eletrônico da 
partícula em mesa vibratória que tenha sido realizado até o momento. Neste contexto, as investigações associadas ao presente trabalho foram realizadas através da aplicação da técnica de sincronização por realimentação de erro (KOLUMBÁN; KENNEDY; CHUA, 1998). A robustez do acoplamento entre sistema mestre e escravo foi verificada considerando modificações nos seguintes parâmetros do circuito eletrônico: amplitude, frequência e fase do sinal oscilante e amplitudes do sinal de tensão DC aplicado.

O trabalho está organizado como segue. $\mathrm{Na}$ seção II, o circuito eletrônico da partícula em mesa vibratória é descrito matematicamente e seu comportamento elétrico é analisado em detalhes. Na seção III, a técnica de sincronização por realimentação de erro é brevemente descrita e aplicada ao circuito eletrônico apresentado na seção II. Aspectos de sincronismo entre os sistema mestre e escravo acoplados são analisados na seção IV, considerando o descasamentos dos seguintes parâmetros: amplitude, frequência, fase do sinal oscilante e amplitude do sinal DC aplicado. Finalmente, as conclusões a respeito das avaliações experimentais são apresentadas na seção V.

\section{Circuito Eletrônico Da Partícula Em Mesa}

\section{Vibratória}

No sistema mecânico da partícula em mesa vibratória da figura 1, uma partícula com massa $m$ colide com uma mesa na direção $x$. A equação que descreve o sistema é dada por:

$$
m \frac{d^{2} x}{d t^{2}}-f\left(x^{*}(t)\right)=-m g
$$

onde $x^{*}(t)=x(t)-s(t) ; s(t)$ é a posição da mesa; $x(t)$ é a posição da partícula; e $g$ é a aceleração gravitacional.
Figura 1 - Sistema mecânico da partícula em mesa vibratória

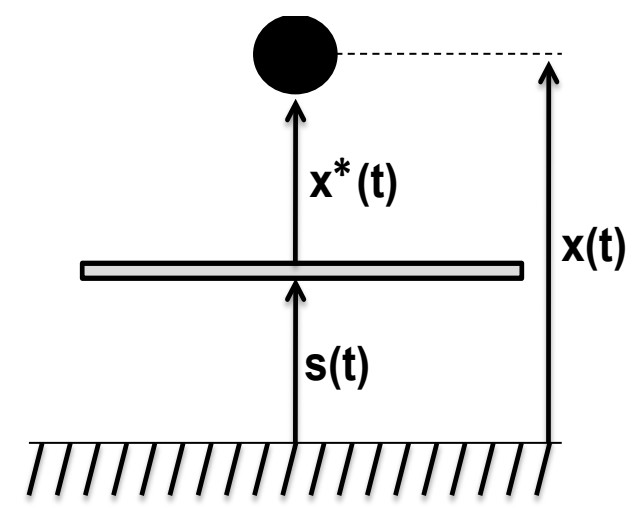

Fonte: o próprio autor

Dependendo dos parâmetros da equação (1), o sistema apresentado pode exibir uma oscilação periódica com período $\mathrm{T}$, passando por sucessivas bifurcações (duplicações de período) com períodos $2 \mathrm{~T}$ e $4 \mathrm{~T}$, até a condição de oscilação caótica não periódica ser atingida. Este sistema pode ser emulado a partir de circuitos eletrônicos conforme ilustrado na figura 2. Este circuito eletrônico representa o comportamento mecânico do sistema da figura 1, permitindo a emulação do comportamento mecânico das colisões de uma partícula sobre uma mesa em movimento. Tal circuito está baseado no circuito eletrônico da partícula em mesa vibratória proposto por Zimmerman e outros (ZIMMERMAN; CELASCHI; NETO, 1992), utilizado para estudar o comportamento dinâmico de uma partícula sobre uma superfície vibrante. No circuito da figura 2 o sinal senoidal $V_{T}=V_{\max } \operatorname{sen}(w t)$ é a entrada do sistema e a equação diferencial que o descreve é dada por:

$$
R_{2} C_{1} C_{2} \frac{d^{2} V_{b}}{d t^{2}}-I_{D}\left(V_{b}, V_{T}\right)=\frac{V_{D}}{R_{1}}
$$

Em (2), o sinal $V_{b}$ corresponde à posição $x$ do sistema da figura 1 , e o sinal $d V_{b} / d t$ corresponde à velocidade $d x / d t$ do mesmo.

Os comportamentos caótico ou periódico podem ser obtidos variando o parâmetro de bifurcação do circuito da figura 2 , que controla a dinâmica 
do sistema. A frequência angular $w$ e a amplitude $V_{\max }$ do sinal senoidal de entrada podem ser considerados como parâmetros de bifurcação deste sistema. O parâmetro de bifurcação $V_{\max }$ foi utilizado para mostrar o comportamento dinâmico do sistema eletrônico da partícula em mesa vibratória. Para a frequência angular fixa de $w=315,6 \mathrm{rad} / \mathrm{s}$ a variação de $V_{\max }$ permite que o sistema oscile periodicamente com período $\mathrm{T}$, passando, novamente, por duplicações sucessivas de período $2 \mathrm{~T}$ e $4 \mathrm{~T}$, até ocorrer uma situação de oscilação não periódica caótica. $\mathrm{O}$ comportamento dinâmico do sistema pode ser analisado observando os diagramas de fase, velocidade da partícula contra posição da mesma. Para o circuito eletrônico, o diagrama de fase observado na tela do osciloscópio é obtido utilizando os respectivos sinais de tensões $V_{c}$ e $V_{b}$ como entradas para as deflexões vertical e horizontal. O comportamento dinâmico do sistema eletrônico da figura 2 com as sucessivas duplicações de período até a situação caótica está ilustrado nos diagramas de fase da figura 3 .

A aplicação de circuitos caóticos muitas vezes requer sincronismo entre sistemas mestre e escravo. Neste caso, torna-se de grande importância a avaliação de técnicas de sincronismo para a aplicação do circuito eletrônico da partícula em mesa vibratória. Na próxima seção, aspectos associados ao sincronismo de sistemas caóticos necessários para a aplicação do circuito em questão são descritos e caracterizados.

\section{Sincronismo Em Sistemas Caóticos}

O sincronismo é muito importante em várias aplicações de sistemas caóticos. Em sistemas de comunicação por exemplo, há dois tipos de deteç̧ão: coerente e não-coerente. $\mathrm{Na}$ detecção coerente cópias exatas das funções base devem ser conhecidas no receptor. Neste caso, há a necessidade de sincronismo entre transmissor e receptor. Já detecção não-coerente não é necessário recuperar as funções base no receptor; neste caso, não é necessário manter sincronismo entre transmissor e receptor, pois em geral a detecção da informação é obtida através da recuperação da envoltória ou comparando-se a energia do sinal detectado com um limiar. Entretanto, é bem conhecido o melhor desempenho atingido pelos sistemas coerentes em relação àqueles com detecção não-coerente, desde que sincronismo entre transmissor e receptor seja atingido.

Figura 2 - Circuito eletrônico da partícula em mesa vibratória baseadoem(ZIMMERMAN;CELASCHI; NETO, 1992). Os valores dos componentes são: resistores em $\mathrm{k} \Omega\left(\mathrm{R}_{1}=1000 ; \mathrm{R}_{2}=10 ; \mathrm{R}=10 ; \mathrm{R}_{\mathrm{f}}=2\right)$, capacitores em $\mathrm{nF} \quad\left(\mathrm{C}_{1}=\mathrm{C}_{2}=\mathrm{C}_{\mathrm{f}}=47\right)$, diodo $\mathrm{D}$ (1N4148), amplificadores operacionais (LM741). Todos os resistores e capacitores utilizados tem tolerância de $\pm 10 \%$ em relação aos seus valores nominais.

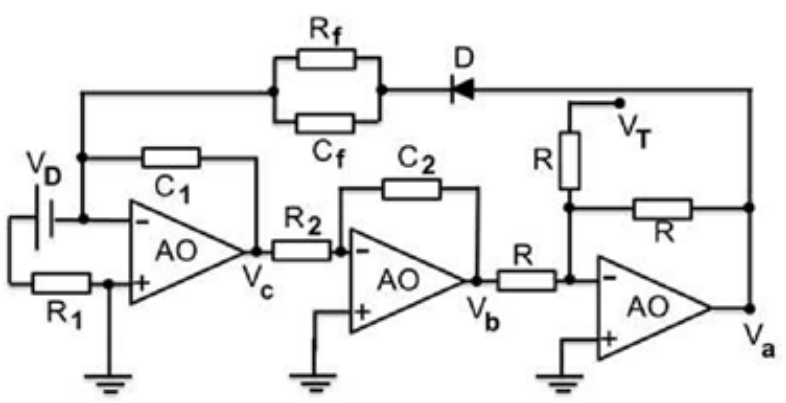

Fonte: o próprio autor

Figura 3 - Diagramas de fase experimentais $\left(\mathrm{V}_{\mathrm{c}} \times \mathrm{V}_{\mathrm{b}}\right)$ para $\mathrm{w}=315,6 \mathrm{rad} / \mathrm{s}$. (a) Período $1, \mathrm{~V}_{\text {max }}=0,62 \mathrm{~V}$; (b) Período 2, $\mathrm{V}_{\text {max }}=0,74 \mathrm{~V}$; (c) Período 4, $\mathrm{V}_{\max }=0,87 \mathrm{~V}$; (d) Comportamento caótico, $\mathrm{V}_{\max }=0,93 \mathrm{~V}$.

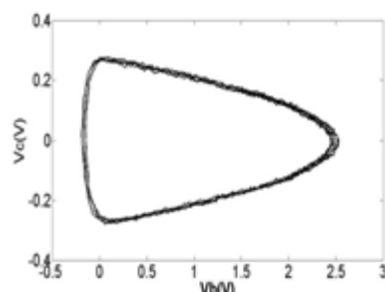

(a)

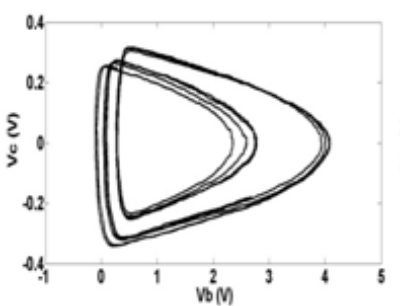

(c)

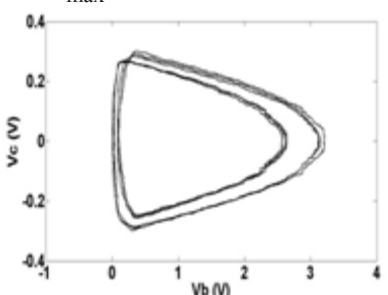

(b)

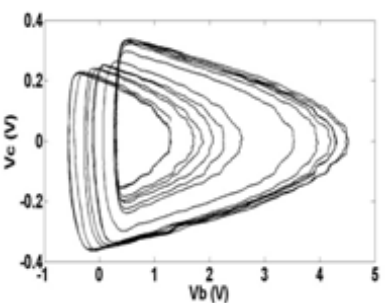

(d)
Fonte: o próprio autor 
Nesta seção, será examinada a técnica de sincronismo utilizada na implementação do sistema proposto. No sistema de sincronização mestreescravo proposto por Pecora e Carroll [11, 16], um sistema dinâmico caótico

$$
g(t)=h(x)
$$

com uma saída escalar $g(t)=h(x)$ é decomposto em dois subsistemas com estados $x_{2}$ e $x_{2}$, respectivamente:

$$
\begin{gathered}
\dot{x}_{1}=f_{1}\left(x_{1}, x_{2}\right) \\
\dot{x}_{2}=f_{2}\left(x_{2}, g(t)\right.
\end{gathered}
$$

sendo $x=\left(x_{1}, x_{2}\right)$ o sinal de saída escalar e, para um sistema de comunicação, $g(t)=h\left(x_{1}(t) x_{2}(t)\right.$ é uma função base caótica, sendo $h($.) obtido do sistema mestre.

O sistema é particionado de tal maneira que os expoentes condicionais de Lyapunov (KOLUMBÁN; KENNEDY; CHUA, 1998) do segundo subsistema descrito por (5) sejam negativos. A técnica Pecora-Carroll (PECORA; CARROLL, 1990), (KOLUMBÁN; KENNEDY; CHUA, 1998) pode ser aplicada na equação diferencial ordinária dada por (2), que descreve o circuito da figura 2. A equação (2) pode ser decomposta em dois subsistemas:

$$
\begin{gathered}
\frac{d V_{b d}}{d t}=-V_{c d} R_{2} C_{2} \\
\frac{d V_{c d}}{d t}=\frac{1}{C_{1}}\left[-I_{D}\left(V_{b d}, V_{T d}\right)-\frac{V_{D d}}{R_{1}}\right]
\end{gathered}
$$

As equações (6) e (7) são fisicamente implementadas através do circuito eletrônico da figura 4.
Figura 4 - Sistema mestre da partícula em mesa vibratória. Os valores dos componentes são: resistores em $\mathrm{k} \Omega\left(\mathrm{R}_{1}=1000 ; \mathrm{R}_{2}=10 ; \mathrm{R}=10 ; \mathrm{R}_{\mathrm{f}}=2\right)$, capacitores em $\mathrm{nF} \quad\left(\mathrm{C}_{1}=\mathrm{C}_{2}=\mathrm{C}_{\mathrm{f}}=47\right)$, diodo $\mathrm{D}$ (1N4148), amplificadores operacionais (LM741). Tolerância dos resistores e capacitores: $\pm 10 \%$ em relação aos valores nominais.

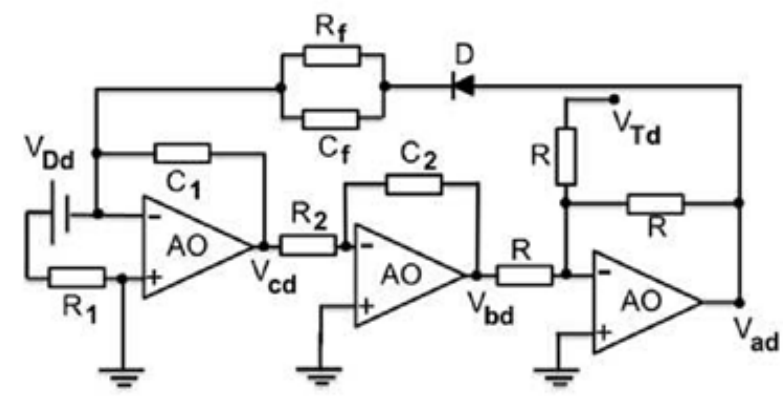

Fonte: o próprio autor

Em termos de um sistema de comunicação, o sistema mestre descrito por (3) gera um sinal de comportamento caótico com função base $g(t)=V_{d}$ , a qual é a saída transmitida. Após passar pelo canal de comunicação, o sinal no receptor $g(t)=V_{d}$ é recebido de forma ruidosa e distorcida, sendo descrito como $r(t)=V_{d l}+\eta(t)$, onde $\eta(t)$ é o ruído branco Gaussiano aditivo com variância $\sigma_{n}^{2}$.

\section{Sincronização por Realimentação de Erro}

Conforme Kolumbán e outros (KOLUMBÁN; KENNEDY; CHUA, 1998), a sincronização por realimentação de erro é uma técnica onde a diferença instantânea entre o sinal estimado $\hat{g}(t)$ e o sinal recebido $r(t)$ produz um sinal de erro escalar $e(t)=r(t)-\hat{g}(t)$ que modifica o estado do receptor tendo em vista minimizar o erro e portanto maximizar o desempenho. O sistema com sincronização por realimentação de erro é descrito por:

$$
\hat{x}=f(\hat{x})+e(t)
$$

sendo $\hat{g}(t)=h(\hat{x})$ e $e($.$) é uma função apropriada$ para o erro de realimentação. Desta forma, a partir de escolhas apropriadas para $h($.$) e e($.) é possível obter:

$$
\lim _{t \rightarrow \infty}\|\hat{x}(t)-x(t)\|=0
$$


Portanto, se o valor de $\hat{x}$ converge para $x$ , então $\hat{g}(t)$ converge para $g(t)$. A técnica de sincronização por realimentação de erro pode ser aplicada ao circuito eletrônico da partícula em mesa vibratória (ZIMMERMAN; CELASCHI; NETO, 1992). Neste caso, um circuito eletrônico com amplificador operacional é utilizado para gerar o sinal de erro escalar $V_{d}-V_{\boldsymbol{c}}$. Este sinal é aplicado ao sistema resposta, como descrito por (6) e (7), através do resistor $R_{A}$. Desta forma, o sistema com realimentação de erro é descrito por:

$$
\begin{gathered}
\frac{d V_{b r}}{d t}=-V_{c r} R_{2} C_{2} \\
\frac{d V_{c r}}{d t}=-\frac{I_{D r}\left(V_{c r}, V_{T r}\right)}{C_{1}}-\frac{V_{d r}}{R_{1} C_{1}}+\varepsilon\left[V_{c d}-V_{c r}\right]
\end{gathered}
$$

com $\varepsilon=1\left(C_{1} R_{A}\right)$ o parâmetro de acoplamento. Uma possível implementação com circuito eletrônico para tal sistema é ilustrado na figura 5 .

Figura 5 - Sistema escravo com realimentação de erro. Os valores dos componentes são: resistores em $\mathrm{k} \Omega\left(\mathrm{R}_{1}=1000 ; \mathrm{R}_{2}=10 ; \mathrm{R}=10 ; \mathrm{R}_{\mathrm{f}}=2 ; \mathrm{R}_{\mathrm{A}}=0,15\right)$, capacitores em $n F \quad\left(C_{1}=C_{2}=C_{\mathrm{f}}=47\right)$, diodo $D$ (1N4148), amplificadores operacionais (LM741). Todos os resistores e capacitores utilizados têm tolerância de $\pm 10 \%$ em relação aos seus valores nominais.

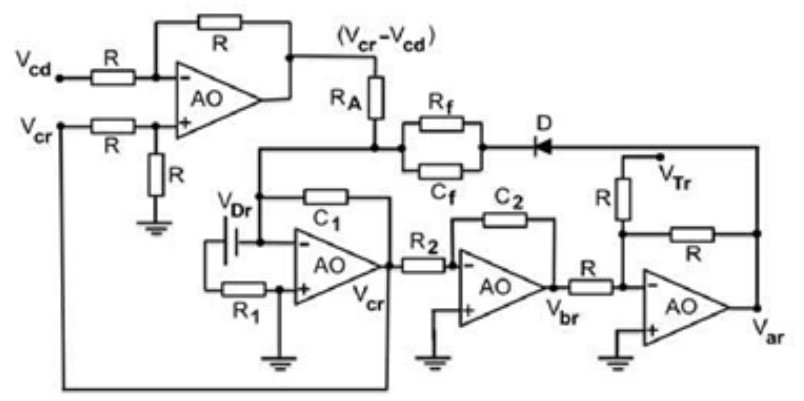

Fonte: o próprio autor

Na figura 6(a) é mostrada a série temporal das tensões de saída dos circuitos mestre e escravo para $R_{A}$ para uma situação de circuito aberto, ou seja, impedância infinita. Neste caso, é normal a ocorrência de falhas no sincronismo entre circuitos mestre e escravo, em que ambos sinais caóticos são gerados independentemente. O comportamento dos sistemas pode ser observado no espaço de fase ( $V_{b d}$ versus $V_{b r}$ ) ilustrado pela figura 6(b). Nele é possível constatar que $V_{b d}$ e $V_{b r}$ não estão sincronizados completamente. Adotando o valor de $150 \Omega$ para $R_{A}$ nota-se pelas figuras 6(c-d) que os circuitos mestre e escravo oscilam em sincronismo. Torna-se evidente que o parâmetro $R_{A}$ é capaz de controlar o acoplamento entre os sistemas mestre e escravo.

Figura 6 - Análise do acoplamento entre os circuitos mestre e escravo para $\mathrm{w}_{\mathrm{d}}=\mathrm{w}_{\mathrm{r}}=315,69 \mathrm{rad} / \mathrm{s}$, $\mathrm{A}_{\mathrm{d}}=\mathrm{A}_{\mathrm{r}}=0,83 \mathrm{~V}$ e $\varphi_{\mathrm{d}} \neq \varphi_{\mathrm{r} \text {. }}$ (a) Série temporal $\mathrm{V}_{\mathrm{bd}}$ e $\mathrm{V}_{\mathrm{br}}$. (b) Trajetória no espaço de fase $\mathrm{V}_{\mathrm{bd}}$ versus $\mathrm{V}_{\mathrm{br}}$ para a situação de desacoplamento $\left(\mathrm{R}_{\mathrm{A}}\right.$ é um circuito aberto); (c) e (d) Mesmos gráficos para a situação de acoplamento $\left(\mathrm{R}_{\mathrm{A}}=150 \Omega\right)$.

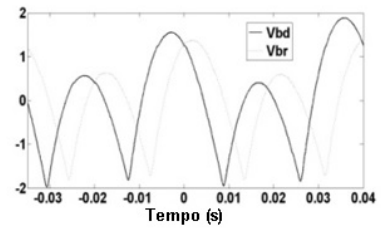

(a)

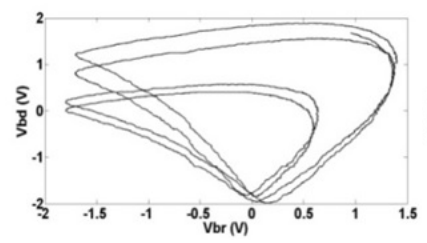

(c)

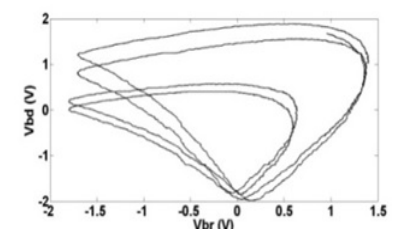

(b)

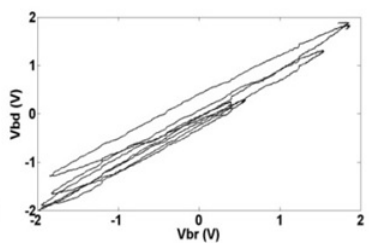

(d)
Fonte: o próprio autor

Geralmente, em sistemas de comunicação coerentes os sistemas transmissor e receptor devem estar em sincronismo. Sejam os sistemas transmissor e receptor representados, respectivamente, pelos circuitos mestre e escravo. Desta forma, é importante investigar a robustez do sincronismo entre os sistemas mestre e escravo em relação ao descasamento de parâmetros. Os parâmetros dos sinais DC $\left(V_{D d}, V_{D r}\right)$ e dos sinais $\mathrm{AC}\left(V_{T d}, V_{T r}\right)$ são os 
mais importantes a serem investigados. Na próxima seção a robustez do desacoplamento entre sistemas mestre e escravo será analisada através da avaliação do sincronismo entre eles perante o descasamento dos parâmetros dos sinais DC e AC.

\section{Robustez Do Acoplamento Com O Circuito Eletrônico Da Partícula Em Mesa Vibratória}

O sincronismo entre dois sistemas caóticos idênticos e independentes, que não dependem de sinal forçante dependente do tempo foi investigado por Pecora e Carroll (PECORA; CARROLL, 1990). $\mathrm{O}$ circuito eletrônico da partícula em mesa vibratória tem um sinal forçante senoidal, caracterizando um sistema caótico não autônomo, ou seja, ele tem um dependência explícita do tempo. Para que o sistema escravo recupere o sinal enviado pelo sistema mestre, ambos sistemas devem possuir casamento de parâmetros para os sinais oscilantes. Fisicamente, alguns parâmetros do sinal senoidal forçante no sistema escravo podem ser diferentes do sinal senoidal forçante no sistema mestre. Como consequência, dependendo do valor do parâmetro de acoplamento entre os sistemas, não será possível estabelecer sincronismo entre ambos (PECORA; CARROLL, 1990).

O circuito eletrônico da partícula em mesa vibratória pode ser utilizado num sistema de comunicação coerente como ilustrado na figura 7 . O transmissor está associado ao sistema mestre da figura 4 e o receptor ao sistema escravo da figura 5. O desempenho do sistema ilustrado na figura 7 depende do sincronismo entre os sistemas transmissor e receptor, o qual depende do casamento de parâmetros DC dos sinais $\left(V_{D d}\right.$ e $\left.V_{D r}\right)$ e AC dos sinais $\left(V_{T d}\right.$ e $\left.V_{T r}\right)$. Nesta seção, o circuito eletrônico da partícula em mesa vibratória será analisado em situações de descasamento dos parâmetros mencionados.
Figura 7 - Diagrama em blocos de um sistema de comunicação coerente que utiliza o circuito eletrônico da partícula em mesa vibratória. O bloco transmissor corresponde ao circuito mestre da figura 4. O bloco receptor corresponde ao circuito escravo da figura 5. O bloco do canal de transmissão é um meio em que é adicionado ruído branco Gaussiano.

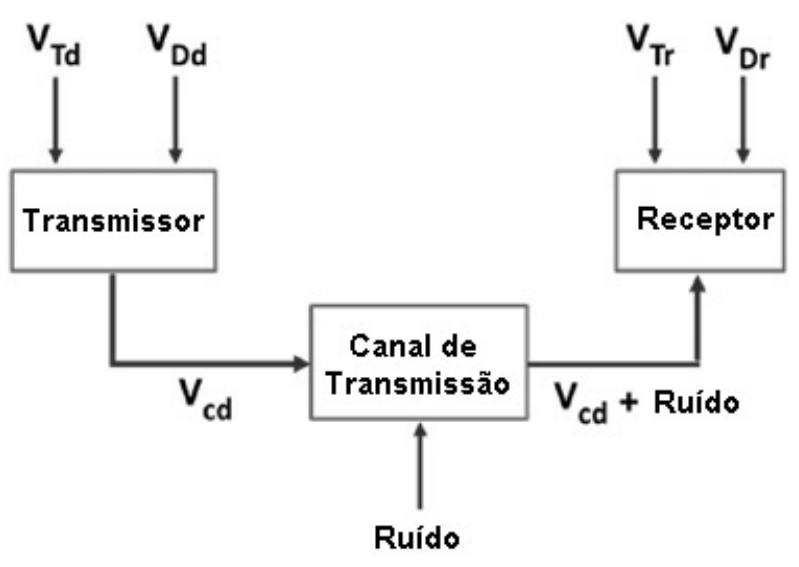

Fonte: o próprio autor

Investigações sobre o sincronismo do sistema da figura 7 foram realizadas considerando o descasamento de parâmetros entre os sinais $V_{\mathbb{Z}}=A_{d} \operatorname{sen}\left(w_{d} t+\varphi_{d}\right) \quad$ e $\quad V_{F}=A_{r} \operatorname{sen}\left(w_{r} t+\varphi_{r}\right)$ adotando como parâmetro de acoplamento $R_{A}=150 \Omega$. Alguns resultados experimentais são apresentados na figura 8 para os seguintes valores de parâmetros: $w_{d}=w_{r}=309,76 \mathrm{rad} / \mathrm{s}, A_{d}=0,63 \mathrm{~V}$ $, 1,1 \mathrm{mV}<A_{r}<3,3 V ; \varphi_{d} \neq \varphi_{r}$. O mecanismo de deslocamento de fase entre os sistemas transmissor e receptor foi implementado utilizando um deslocador de fase eletrônico e o valor do parâmetro de fase foi variado ao longo de uma faixa de valores durante a análise experimental. É interessante observar que o sincronismo foi mantido entre transmissor e receptor em todos os casos. Também nota-se que na presença de descasamento do parâmetro amplitude entre os sinais oscilantes dos circuitos transmissor e receptor sempre há sincronização generalizada (PIKOVSKY, 1984). Na figura 8(a) é possível notar que $V_{b d}$ e $V_{b r}$ não são idênticos, mas o sistema receptor segue o sistema transmissor 
com um pequeno erro. Já no gráfico da figura 8(b) é possível notar sincronização generalizada $\left(V_{b d}\right.$ aproximadamente igual a $V_{b r}$ ) por um longo período de tempo. Destas avaliações experimentais, concluise que os sistemas permanecem em sincronismo mesmo com um grande descasamento do parâmetro amplitude entre transmissor e receptor.

Figura 8 - Séries temporais do processo de sincronização experimental para o descasamento de parâmetros entre $V_{\mathbb{Z}}=A_{d} \operatorname{sen}\left(w_{d} t+\varphi_{d}\right) \quad \mathrm{e}$ $V_{T}=A_{r} \operatorname{sen}\left(w_{r} t+\varphi_{r}\right)$. Parâmetros adotados: $A_{d}=0,63 \mathrm{~V}, \quad A_{r}$ na faixa $1,1 \mathrm{mV}<A_{r}<3,3 \mathrm{~V}$, $w_{d}=w_{r}=309,76 \mathrm{rad} / \mathrm{s}, R_{A}=150 \Omega$ e $R_{A}=150 \Omega$.

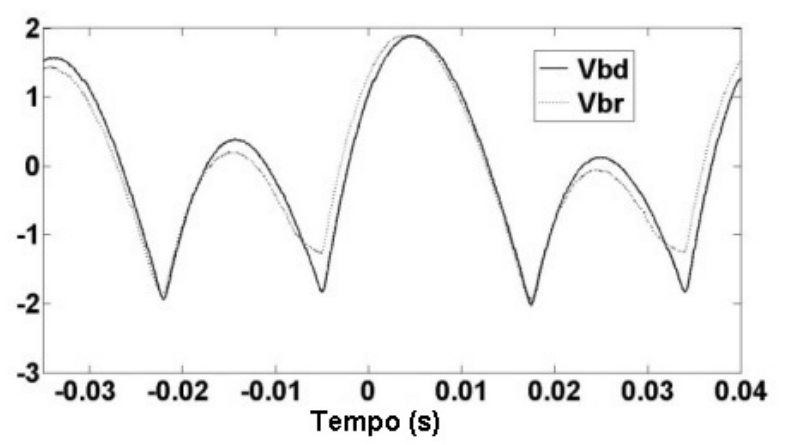

(a)

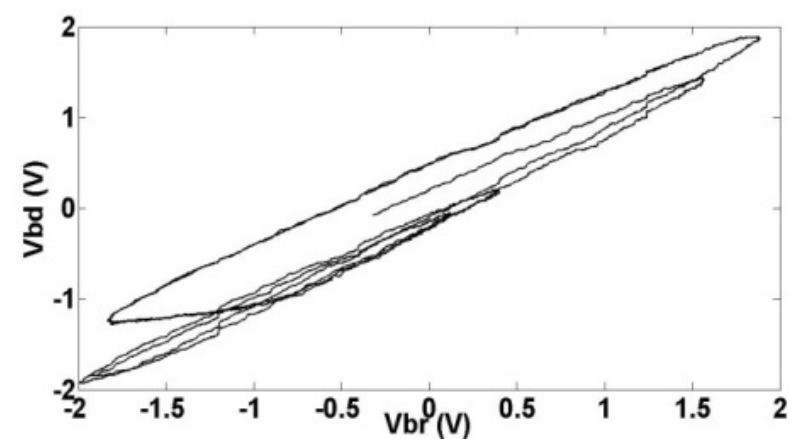

(b)

Fonte: o próprio autor

Por sua vez, o atraso entre os sinais oscilantes dos sistemas não afeta o sincronismo entre os sistemas. $\mathrm{Na}$ figura 9 são apresentados alguns resultados experimentais considerando os seguintes valores adotados para os parâmetros: $A_{d}=A_{r}=0,63 \mathrm{~V}, \varphi_{d} \neq \varphi_{r}$
, $w_{d}=309,76 \mathrm{rad} / \mathrm{s}$ e $3,8 \mathrm{rad} / \mathrm{s}<w_{r}<14,7 \times 10^{6} \mathrm{rad} / \mathrm{s}$ . Pode ser verificado na figura 9(a) que os sinais do transmissor e receptor estão deslocados por um nível DC que poderia ser removido utilizando um filtro. Novamente, o descasamento entre os sinais oscilantes do transmissor e receptor não afetaram o sincronismo entre eles. Além disso, é possível verificar que o sincronismo é mantido mesmo para um grande descasamento do parâmetro frequência entre os sistemas.

Figura 9 - Séries temporais do processo de sincronização experimental para o descasamento de parâmetros entre $V_{T d}=A_{d} \operatorname{sen}\left(w_{d} t+\phi_{d}\right) \quad$ e $\quad V_{T r}=A_{r} \operatorname{sen}\left(w_{r} t+\phi_{r}\right)$ . Parâmetros adotados: $A_{d}=A_{r}=0,63 V, \varphi_{d} \neq \varphi_{r}$ e $3,8 \mathrm{rad} / \mathrm{s}<A_{r}<14,7 \times 10^{6} \mathrm{rad} / \mathrm{s}$ e $R_{A}=150 \Omega$.

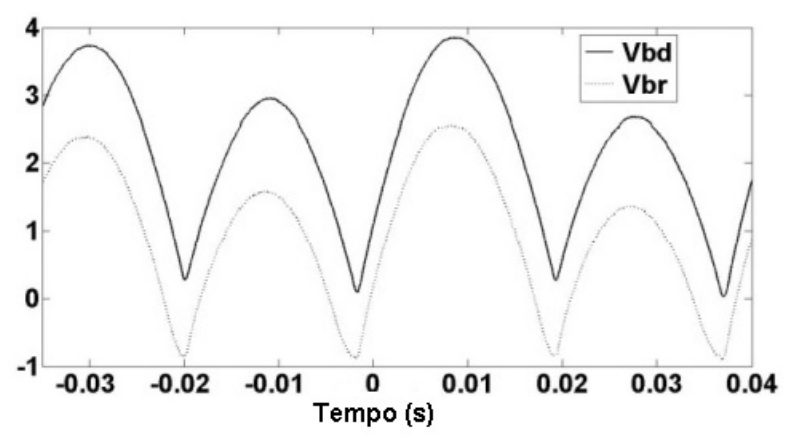

(a)

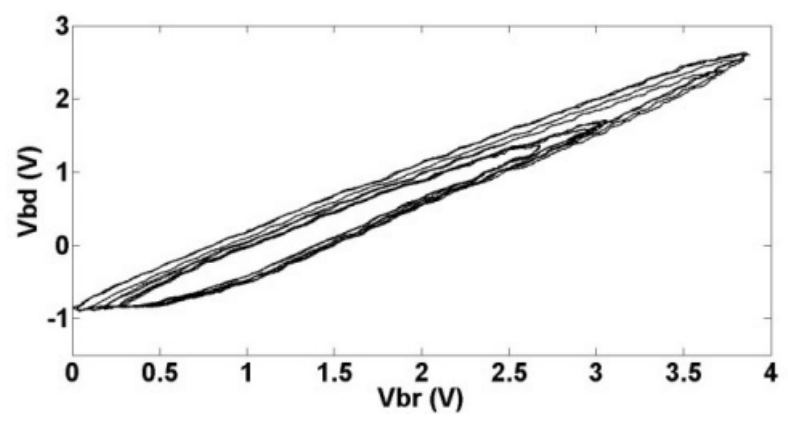

(b)

Fonte: o próprio autor

Os próximos resultados experimentais ilustram a robustez do sincronismo entre os sistemas para a situação de descasamento dos sinais DC. Na figura 10 está ilustrada a característica de sincronismo 
entre os sistemas mestre e escravo para as seguintes condições: descasamento dos sinais DC $\left(V_{D d}\right.$ e $V_{D r}$ ) e descasamento de fase $\left(\varphi_{d} \neq \varphi_{r}\right)$ entre os sinais forçantes $V_{T d}$ e $V_{T r}$. Nesta avaliação é possível notar que os sistemas estão em sincronismo mas os sinais caóticos têm diferentes amplitudes, ou seja, há uma sincronização generalizada (PIKOVSKY, 1984).

Figura 10 - Séries temporais do processo de sincronização experimental para o descasamento de parâmetros entre os sinais DC ( $V_{D d}$ e $\left.V_{D r}\right)$. Parâmetros adotados: $V_{D d}=1,5 V, 0,6 V<V_{D r}<3,61 V$ $, \quad w_{d}=w_{r}=310,1 \mathrm{rad} / \mathrm{s}, \quad A_{d}=A_{r}=0,53 \mathrm{~V}, \varphi_{d} \neq \varphi_{r} \quad \mathrm{e}$ $R_{A}=150 \Omega$.

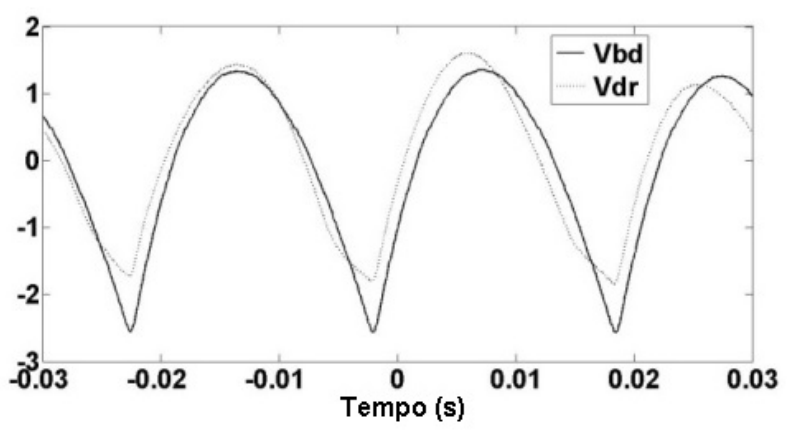

(a)

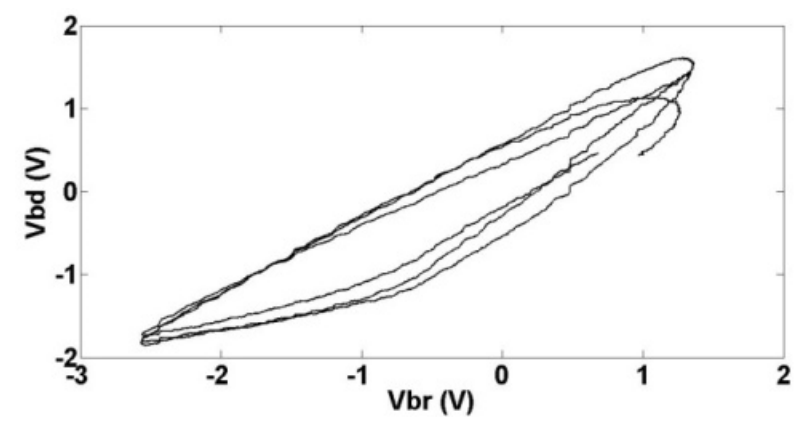

(b)

Fonte: o próprio autor

\section{Conclusões}

Testes experimentais de sincronismo considerando acoplamento com $R_{A}=150 \Omega$ foram realizados para situações de descasamento de parâmetros (sinais DC e AC) entre sistemas transmissor e receptor. O acoplamento entre os sistemas foi implementado utilizando a técnica de sincronismo por realimentação de erro. As figuras 8, 9 e 10 mostram a robustez do acoplamento quanto ao descasamento dos parâmetros dos sinais DC. Nesta situação sempre é possível manter algum tipo de sincronismo entre os sistemas: sincronização generalizada ou sincronização de fase. Esta característica é de vital importância na implementação de sistemas de comunicação com detecção coerente. Em sistemas de telecomunicações convencionais é sempre necessário um circuito para manter sincronismo entre os sistemas transmissor e receptor, conhecido como fase de malha fechada (PLL - Phase Locked Loop). É interessante observar que os sistemas transmissor e receptor aqui analisados sempre mantêm sincronismo e, desta forma, não necessitariam de um circuito PLL.

Portanto, o circuito eletrônico da partícula em mesa vibratória se devidamente acoplado pode ser empregado na implementação de sistemas de comunicação coerentes. Trabalhos futuros pretendem aplicar o circuito eletrônico da partícula em mesa vibratória em sistemas de telecomunicações para aplicações de criptografia e modulação caótica.

\section{Referência}

BOCHENG, B.; ZHONG, L.; JIANPING, X., New chaotic system and its hyperchaos generation, Journal of Systems Engineering and Electronics, v. 20, n. 6, p. 1179,1187, Dec. 2009.

CHUA, L. O.; ITHOH, M.; KOCAREV, L.; ECKERT, K. Chaos synchronization in Chua's circuit. Journal of Circuits, Systems and Computers, v. 3, n. 1, p. 93-108, 1993.

CUOMO, K. M.; OPPENHEIM, A. V.; STROGATS, S. H. Synchronization of Lorenz-based chaotic circuits with applications to communications. IEEE Trans. Circuist Syst. II, Analog Digit. Signal Process., v. 40, n. 10, p. 626-633, 1993. 
FRADKOV, A. L.; ANDRIEVSKY, B.; EVANS, R. J., Adaptive Observer-Based Synchronization of Chaotic Systems With First-Order Coder in the Presence of Information Constraints. IEEE Transactions on Circuits and Systems I: Regular Papers, v. 55, n. 6, p. 1685-1694, July 2008.

FRADKOV, A. L.; ANDRIEVSKY, B.; EVANS, R. J.,. Synchronization of Passifiable Lurie Systems Via Limited-Capacity Communication Channel. IEEE Transactions on Circuits and Systems I: Regular Papers, v. 56, n. .2, p. 430-439, Feb. 2009.

HE, W.; QIAN, F.; HAN, Q-L.; CAO, J. Lag QuasiSynchronization of Coupled Delayed Systems With Parameter Mismatch. IEEE Transactions on Circuits and Systems I: Regular Papers, v. 58, n. 6, p. 1345-1357, June 2011.

HUANG, Y.; ZHANG, P.; ZHAO, W., Novel Grid Multiwing Butterfly Chaotic Attractors and Their Circuit Design. IEEE Transactions on Circuits and Systems II: Express Briefs. v. 62, n. 5, p. 496-500, May 2015.

JOSEPH, S. K.; MARIÑO, I. P.; SANJUÁN, M. A. F. Effect of the phase on the dynamics of a perturbed bouncing ball system. Commun. Nonlinear Sci Numer. Simulat., v. 17, p. 3279-3286, 2012.

KOLUMBÁN, G.; KENNEDY, M. P.; CHUA, L. O. The role of synchronization in digital communications using chaos - Part II: Chaotic modulation and chaotic synchronization. IEEE Trans. Circuits Syst. I: Theory Appl., v. 45, n. 1, p. 1129-1140, 1998.

LI, C.; SPROTT, J. C.; THIO, W.; ZHU, H. A New Piecewise Linear Hyperchaotic Circuit. IEEE Transactions on Circuits and Systems II: Express Brief, v. 61, n. 12, p. 977-981, Dec. 2014.

LI, G.; JIN, G.; CHEN, X. A butterfly chaotic attractor and its circuit implementation. In: INTERNATIONAL CONFERENCE ON INFORMATION SCIENCE, ELECTRONICS AND ELECTRICAL ENGINEERING (ISEEE'14). 2014. Anais... Sapporo-Japão, 2014. v. 3, p. 1906,1907.

LI, Z.; LI, K; WEN, C.; SOH, Y. C., A new chaotic secure communication system. IEEE Transactions on Communications, v. 51, n. 8, p. 1306-1312, Aug. 2003.

LIANG, X.; ZHANG, J.; XIA, X. Improving the Security of Chaotic Synchronization With a Delta-Modulated Cryptographic Technique. IEEE Transactions on Circuits and Systems II: Express Briefs, v. 55, n. 7, p. 680,684, July 2008.

MELlO, T. M.; TUFILlARO, N. B. Strange attractors of a bouncing ball. American Journal of Physics, v. 55, n. 4, p. 316-320, 1987.

PECORA, L. M.; CARROLL, T. L. Synchronization in chaotic systems. Phys. Rev. Lett., v. 64, p. 821824, 1990.

PFALTZGRAFF, D. J. Analog simulation of the bouncing-ball problem. American Journal of Physics, v. 37, n. 10, p. 1008-1013, 1969.

PIKOVSKY, A. S. On in the interaction of strange atractors. Z. Phys. B-Condensed Matter, v. 55, p. 149-154, 1984.

PISARCHIK, A. N.; RUIZ-OLIVERAS, F. R. Optical chaotic communication using generalized and complete synchronization. IEEE Journal of Quantum Electronics, v. 46, n. 3, p. 279-284, March 2010.

PIZOLATO JUNIOR, J. C.; ROMERO, M. A.; NETO, L. G. Chaotic communication based on the particle-ina-box electronic circuit. IEEE Transactions on Circuits and Systems - I: regular papers, v. 55, n. 4, p. 1108-1115, 2008.

REIST, P.; D'ANDREA, R. Control of nonlinear systems with symmetries using chaos. In: IEEE 51ST ANNUAL CONFERENCE ON DECISION AND CONTROL (CDC), 2012. Anais... Maui (Hawaii)-USA, 2012. p. 997-1002.

ROSENBLUM, M. G.; PIKOVSKY, A. S.; KURTHS, J. Phase synchronizations effects in a lattice of nonidentical Rössler oscillators, Phys. Review E, v. 55, n. 3, p. 23532361, 1997.

RULKOV, N. F.; SUSHCHIK, M. M.; TSIMRING, 
L. S.; ABARBANEL, H. D. I. Generalized synchronizations of chaos in directionally coupled chaotic systems. Phys. Rev. E, v. 51, p. 980-994, 1995.

SPROTT, J. C. A new class of chaotic circuit. Physics Letters A, v. 266, p. 19-23, 2000.

SPROTT, J. C. A new chaotic Jerk circuit. IEEE Transactions on Circuits and Systems - II: Express Briefs, v. 58, n. 4, p. 240-243, 2011.

SRISUCHINWONG, B.; SUKJARERNCHAIKUL, P., Twin Current-Tunable Chaotic Jerk Equations in Two Differently Swapped-Pair. In: INTERNATIONAL SYMPOSIUM ON CIRCUITS. COMPUTER, CONSUMER AND CONTROL (IS3C), 2014. Anais...Taichung-Taiwan, 2014. p. 749-752.

SUNEEL, M. Electronic circuit realization of the logistic map. Sãdhanã, v. 31, n. 1, p. 69-78, 2006.

TUFIllaro, N. B.; AlBANO, A. M. Chaotic dynamics of a bouncing ball. American Journal of Physics, v. 54, p. 939-944, 1986.

VINCENT, T. L. Controlling a ball to bounce at a fixed height. In: PROCEEDINGS OF THE AMERICAN CONTROL CONFERENCE, 1995. Anais...Seattle(Washington)-USA, 1995. v. 1, p. 842,846 .

WU, Z-G.; SHI, P; SU, H.; CHU, J. SampledData Synchronization of Chaotic Lurie Systems With Time Delays. IEEE Transactions on Neural Networks and Learning Systems, v. 24, n. 3, p. 410421, March 2013.

ZIMMERMAN, R. L.; CELASCHI, S.; NETO, L.

G. The electronic bouncing ball. American Journal of Physics, v. 60, n. 4, p. 370-375, 1992. 
